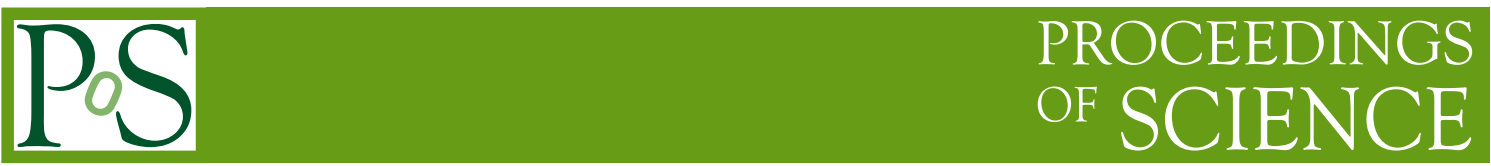

\title{
Gamma-ray burst observations with the CALET Gamma-ray Burst Monitor
}

\author{
Yuta Kawakubo* \\ Department Physics and Astronomy, Louisiana State University \\ E-mail: kawakubol@lsu.edu
}

\section{for the CALET collaboration ${ }^{\dagger}$}

The CALET Gamma-ray Burst Monitor (CGBM) is a secondary scientific instrument of the CALET. The CGBM has been monitoring X-ray and gamma-ray sky since October 2015. The main observational target of the CGBM is a gamma-ray burst (GRB). The CGBM can observe GRB light curves and spectra in the energy range from $7 \mathrm{keV}$ to $20 \mathrm{MeV}$ thanks to two kinds of scintillation detectors which are the Hard X-ray Monitor (HXM) and the Soft Gamma-ray Monitor (SGM). As of the end of June 2019, CGBM has detected 161 GRBs since October 2015. The durations of GRBs detected by the CGBM were measured by the SGM in the $40 \sim 1000 \mathrm{keV}$ energy band. As a result, 19 out of 161 GRBs were short bursts which are a primary candidate of the electromagnetic counterparts of gravitational wave source. We performed spectral analysis for bright 4 short GRBs out of 19. We found spectral parameters of the 4 GRBs were consistent with an expectation by the synchrotron shock model. Although observed fluxes of the 4 GRB in the $30 \sim 1000 \mathrm{keV}$ range were more than $\sim 10$ times as large as GRB 170817A, $E_{\text {peak }}$ of GRB 180703B was similar to that of GRB 170817A.

36th International Cosmic Ray Conference -ICRC2019-

July 24th - August 1st, 2019

Madison, WI, U.S.A.

\footnotetext{
* Speaker.

${ }^{\dagger}$ for collaboration list see PoS(ICRC2019)1177
} 


\section{Introduction}

A Gamma-ray burst (GRB) is the brightest phenomenon which emits enormous energy in a short time in the form of electromagnetic waves [1] . A primary emission of GRB is called a prompt emission. A prompt emission is bright and variable in X-rays and gamma-rays. The typical time scale of variability of prompt emission is $\sim 10^{-3} \mathrm{~s}$. The duration distribution of prompt emission is bimodal distribution in the log scale [2]. A long GRB, of which duration is longer than $2 \mathrm{~s}$, and a short GRB, of which duration is less than $2 \mathrm{~s}$, seem to have different origins. Since some of the long bursts are associated with supernovae, long bursts seem to be caused by collapses of massive stars [3]. Short bursts seem to be caused by mergers of binary neutron stars or a neutron star and a black hole. GRB 170817A, which is the first short GRB associated with the gravitational wave event from the binary neutron star merger, was detected by Fermi-GBM and INTEGRAL SPI-ACS $[4,5]$. The observation of GRB $170817 \mathrm{~A}$ and GW170817 is an evidence that binary neutron star mergers can cause short GRBs.

GRB 170817A is only one GRB associated with a gravitational wave source so far. Further observations of short GRBs are needed to confirm the association between short GRBs and binary neutron star mergers. Also, observations of short GRBs associated with gravitational waves can be a clue to understanding the environment around GRB site. LIGO \& Virgo started the third observation run in April 2019 after the major upgrades. Additional detections of electromagnetic counterparts have increased in importance as a result.

The CALorimetric Electron Telescope (CALET) is a payload instrument on the Japan Experimental Module (JEM) on the International Space Station (ISS) [6]. CALET has been observing cosmic electron, proton, and gamma-rays since October 2015. CALET Gamma-ray Burst Monitor (CGBM), which is the secondary scientific instrument of CALET, has been monitoring the occurrence of GRBs and observing light curves and spectra of GRB since the observation started [7]. The energy range of CGBM from $7 \mathrm{keV}$ to $20 \mathrm{MeV}$ covers the typical energy range of GRB prompt emission. In this paper, we will present an overview of GRB observations in three years and nine months with CGBM. Also, we will present spectral analysis for 4 bright short GRBs detected by CGBM because a short GRB is a primary candidate of the electromagnetic counterparts of binary neutron star mergers. Detail of gamma-ray observations of CAL will be presented in different paper. All quoted errors in this paper correspond to $90 \%$ confidence level.

\section{CALET Gamma-ray Burst Monitor (CGBM)}

Figiure 1 is a schematic image of CALET. CGBM is mounted on the upper side of the CALET. CGBM consists of two Hard X-ray Monitors (HXMs) and one Soft Gamma-ray Monitor (SGM). Both the HXM and SGM are scintillation detectors. Detector characteristics are summarized in Table 1. The crystals of the HXM and SGM are $\mathrm{LaBr}_{3}(\mathrm{Ce})$ and BGO, respectively. Since $\mathrm{LaBr}_{3}(\mathrm{Ce})$ has a larger light yield than $\mathrm{NaI}(\mathrm{Tl})$ crystal, $\mathrm{HXM}$ covers the energy range from $7 \mathrm{keV}$ to $1 \mathrm{MeV}$ for X-rays and gamma-rays. HXM has a Be entrance window, of which thickness is $410 \mu m$, for low energy X-rays. Thanks to the high stopping power of BGO, SGM covers the energy range from $40 \mathrm{keV}$ to $20 \mathrm{MeV}$ for X-rays and gamma-rays. More detailed information is described in Yamaoka et al. (2013) [7] 


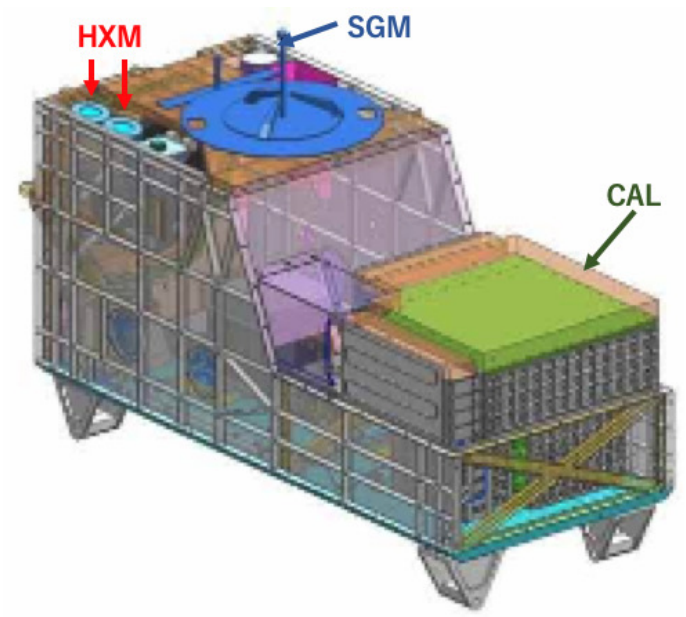

Figure 1: Schematic image of CALET. Two HXMs and one SGM are installed at the upper side of CALET.

\begin{tabular}{ccc}
\hline & HXM & SGM \\
\hline \hline Sensor & $\mathrm{LaBr}_{3}(\mathrm{Ce})+$ photomultiplier tube & BGO + photomultiplier tube \\
Number of detector & 2 & 1 \\
Energy range & $7 \mathrm{keV} \sim 1 \mathrm{MeV}$ & $40 \mathrm{keV} \sim 20 \mathrm{MeV}$ \\
Diameter of crystal & $66.1 \mathrm{~mm}$ (small diameter) $78.7 \mathrm{~mm}$ (large diameter) & $101.6 \mathrm{~mm}$ \\
Thickness of crystal & $12.7 \mathrm{~mm}$ & $76 \mathrm{~mm}$ \\
Field of view & $\sim 3 \mathrm{sr}$. & $\sim 8 \mathrm{sr}$. \\
\hline
\end{tabular}

Table 1: CGBM characteristics

CGBM data types are summarized in Table 2. CGBM is always collecting the monitor data, which include time history $(\mathrm{TH})$ data and pulse height $(\mathrm{PH})$ data. If the CGBM onboard trigger system detects a transient event, CGBM captures the event data automatically. For a broad dynamic range, the output of each sensor is divided into two signals, and each signal is connected to amplifiers with different gains before being fed to the Analog-to-digital converter (ADC). Therefore, monitor data and event data have 2 sets of data which are called High gain data and Low gain data, respectively. The difference between High gain and Low gain is $\sim 30$. TH data are light curve data with $1 / 8 \mathrm{~s}$ time resolution and 4 (High gain) +4 (Low gain) energy channels. PH data are spectrum data with $4 \mathrm{~s}$ time resolution and 102 (High gain) +410 (Low gain) energy channels. Event data are time-tagged data with time resolution $62.5 \mu$ s and 4096 (High gain) +4096 (Low gain) energy channels. Also, periodic data, which include housekeeping data, are collected every second.

\section{Summary of GRB observation with CGBM}

As of the end of June 2019, CGBM has detected 161 GRBs, which were confirmed by other instruments, since October 5, 2015. The detection rate is $\sim 43$ GRBs per year with $\sim 60 \%$ duty cycle, because CGBM high voltages are turned off at the high latitude regions and around the South Atlantic Anomaly to protect photomultiplier tubes from extreme high count rates due to 


\begin{tabular}{ccccc}
\hline & Time history (TH) & Pulse height (PH) & Event & Periodic \\
\hline \hline Time resolution & $0.125 \mathrm{~s}$ & $4 \mathrm{~s}$ & $62.5 \mu \mathrm{s}$ & $1 \mathrm{~s}$ \\
Channel (High-gain) & 4 & 102 & 4095 & - \\
Channel (Low-gain) & 4 & 410 & 4095 & - \\
Collection timing & Anytime & Anytime & Only when onboard triggers occur & Anytime \\
\hline
\end{tabular}

Table 2: Data types

increased charged particle backgrounds. Figure 2 shows the $T_{90}$ duration distribution of CGBMdetected GRBs with those of other instruments. Duration of CGBM-detected GRBs measured by SGM in the 40 1000 keV band using 'battblocks', which is a software for Swift-BAT data to measure the duration using the Bayesian block method [8]. TH data were used for duration calculation. If TH data are missing or the durations calculated with TH data are shorter than $2 \mathrm{~s}$, duration calculation was performed with event data. The classification of GRBs was performed with $T_{90}$ without considering hardness. The numbers of long GRBs and short GRBs are 142 and 19, respectively. The short GRB fraction in the CGBM GRB sample is $12 \%$. Those of SwiftBAT, Fermi-GBM, and BATSE are $\sim 9 \%, \sim 17 \%$, and $\sim 22 \%$, respectively. CGBM can observe short GRBs which are the primary candidate of electromagnetic counterparts of gravitational wave source.

Figure 3 shows the incident angle distribution of CGBM-detected GRBs. The radial axis is the zenith angle $\theta$ of the SGM. The $\theta=0$ corresponds to the zenith of the SGM. The azimuth $\phi=0$ corresponds to the front direction of CALET (CAL side). The azimuth $\phi$ increases along the counterclockwise direction. Since CGBM has no capability for position determination, GRB position information were referred from the GCN circulars or the GCN notices of Swift-BAT, XRT, UVOT, Fermi-GBM, LAT, MAXI, INTEGRAL or IPN $[9,10]$. There is $5 \sim 15$ deg uncertainty for each point in Figure 3 due to GRB position errors, which depends on the instruments of position source, and CGBM attitude. Since CALET is a payload instrument on the ISS, the ISS structures block some part of the CALET field of view. The fixed ISS structures viewing from the CAL were shown in Figure 3. Aside from the fixed ISS structure, there are moving structures (e.g., robot arms, solar paddles.). Since the position of moving structure depends on the time of the occurrence of the bursts, Figure 3 includes no moving structure. 7 GRBs were detected in blocked region by the ISS structures. We are still investigating influence of scattering and blocking on GRB observations. The maximum of $\theta$ is $115^{\circ}$ for GRB 190531B. Although the ISS structures block some part of the field of view of CGBM, the CGBM can monitor the X-ray and gamma-ray sky with the wide field of view.

\section{Spectral analysis for short GRBs}

CGBM has detected 19 short bursts. We performed spectral analysis for short GRBs detected by CGBM to compare spectral parameters with those of GRB 170817A [5], which is the only GRB coincident with a gravitational wave event due to the binary neutron star merger. We analyzed GRBs which meet all the following conditions.

1. The position was determined by other instruments. 


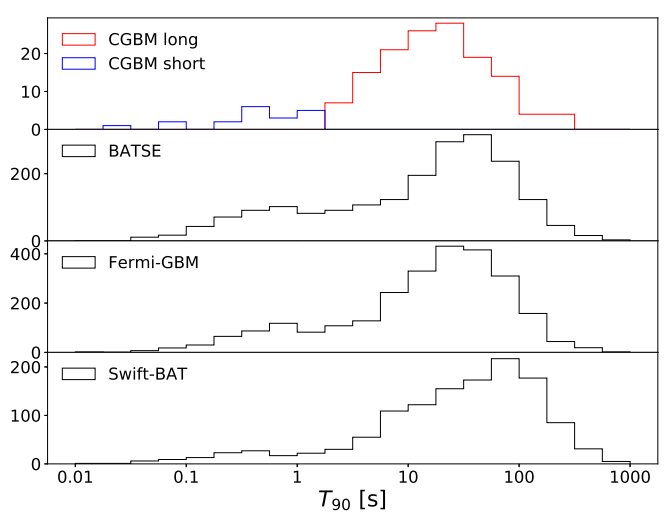

Figure 2: $T_{90}$ distribution of GRBs. The energy ranges of CGBM, BATSE, Fermi-GBM, and Swift-BAT are $40 \sim 1000 \mathrm{keV}, 25 \sim 2000 \mathrm{keV}, 50 \sim 300 \mathrm{keV}$, and $15 \sim 350 \mathrm{keV}$, respectively [11, 12, 13, 14, 15].

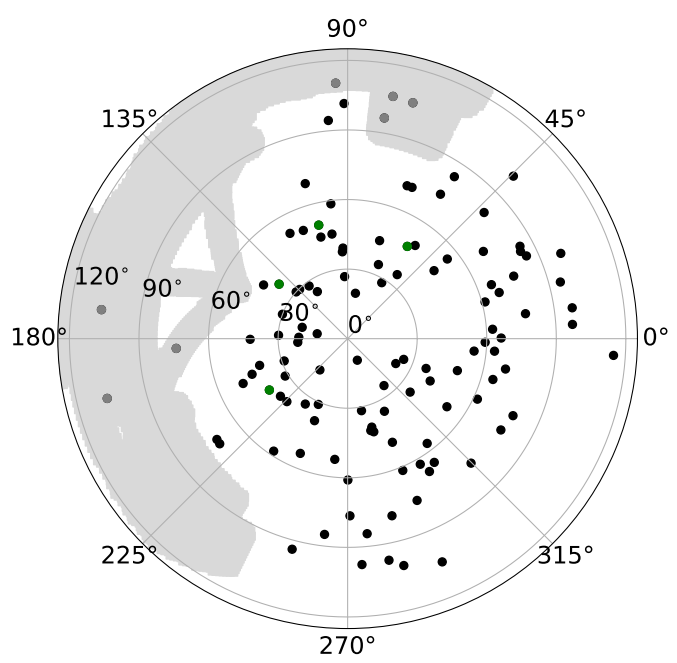

Figure 3: Incident angle distribution of GRBs in the SGM field of view. The radial axis corresponds to $\theta$. Blue points are GRB position in the SGM field of view. Green points show 4 short GRBs which we performed spectral analysis in this paper. A gray shaded region is the ISS fixed structure viewed from CALET. Gray points show 7 GRBs blocked by the ISS structures.

2. The zenith angle of SGM is less than $70 \mathrm{deg}$.

3. The signal was detected by all CGBM detectors.

4. The signal-to-noise ratio (SNR) measured by TH data is greater than 20 .

Since CGBM has no capability of localization of GRBs, the first condition is required. The second and third conditions are needed to avoid large uncertainty of the determined spectral parameters due to the blocking and scattering by ISS structures. The energy response for spectral analysis was calculated using the CGBM simulator based on Geant4 [16]. The CGBM simulator includes material distribution of CGBM detectors and simplified CALET contents and frame. Also, the energy resolution of each detector, which was measured in the ground calibration test, were included. The dependency of gain, energy resolution, and detection efficiency on the incident position of photons was considered for HXM. However, the CGBM simulator was developed without considering the ISS structures because the ISS structures are large and can move. Therefore, we need to exclude from the analysis cases in which the GRB came from large angles. The fourth condition ensures enough statistics for spectral analysis. Four GRBs met the conditions and are listed in Table 3. Also, Four GRBs were shown as green points in Figure 3. Four GRBs are different from the 7 GRBs blocked by the ISS structures.

For spectral analysis, we used event data. A gain correction was applied to event data for each detector by fitting the $511 \mathrm{keV}$ line due to annihilation of positrons in the background spectra. The 
spectrum was generated from event data for the whole burst duration $\left(T_{100}\right)$ measured by SGM in the $40 \sim 1000 \mathrm{keV}$ energy band. Background spectra were generated from the data before and after the burst duration. Then, the two background spectra were summed and averaged. We applied dead time correction to both foreground and background spectra. Since the event data for GRB 160709A ended $\sim 2 \mathrm{~s}$ after the end of the burst duration, the background spectra for GRB 160709A were generated from the data before the burst.

Spectral analysis was performed with XSPEC version 12.10.1 in FTOOLS which was developed by NASA HEASARC [17]. We used a cutoff power-law as a fitting model. The formula is $N(E)=A\left(\frac{E}{200 \mathrm{keV}}\right)^{\alpha} \exp \left[-\frac{E(2+\alpha)}{E_{\text {peak }}}\right]$, where $\alpha$ is a photon index, $E_{\text {peak }}$ is a peak energy in the $v F_{V}$ spectrum, and $A$ is a normalization at $200 \mathrm{keV}$. Considering the uncertainty of the cross-calibration between each detector, the model multiplied by constant factors for each detector. The constant factor of the HXM1 $\left(C_{\mathrm{HXM} 1}\right)$ and the SGM $\left(C_{\mathrm{SGM}}\right)$ are normalized to the constant factor of HXM2 which is assumed to be unity. $C_{\mathrm{HXM} 1}$ and $C_{\mathrm{SGM}}$ tend to be distributed around $\sim 1$ and $\sim 1.2$, respectively [18]. Also, a cross-calibration between CGBM and Swift-BAT was performed in Kawakubo et al. 2018 [19]. Based on the cross-calibration, the flux determined by HXM was consistent with that of Swift-BAT in the $30 \sim 150 \mathrm{keV}$ energy range at least.

As an example of spectral analysis, Figure 4 shows the spectrum of GRB 180703B with the optimized cutoff power-law model. The spectrum of GRB 180703B was consistent with the cutoff power-law model with $\alpha=-1.21_{-0.22}^{+0.24}$ and $E_{\text {peak }}=146_{-17}^{+18} \mathrm{keV}$. Other three GRBs were also analyzed in the same way as GRB 180703B. The derived spectral parameters for 4 GRBs are summarized in Table 3. The spectra of all 4 GRBs are consistent with cutoff power-law models (null hypothesis probability $>0.1$ ). $C_{\mathrm{HXM} 1}$ and $C_{\mathrm{SGM}}$ of $4 \mathrm{GRBs}$ are consistent with $\sim 1$ and $\sim 1.2$, respectively. The $\alpha$ of 4 GRBs are consistent with the photon index expected by synchrotron shock model [20]. The energy fluxes in the $30 \sim 1000 \mathrm{keV}$ for the 4 GRBs were calculated and shown in Table 3.

Figure 5 shows the distribution of energy flux and $E_{\text {peak }}$. Although there was no significant signal around the GRB 170817A in the CGBM data, GRB 170817A was also plotted in Figure 5 based on Fermi-GBM observations [21]. Figure 5 shows the fluxes of 4 GRBs are more than $\sim 10$ times as bright as GRB 170817A. However, Figure 5 also shows $E_{\text {peak }}$ of GRB 180703B was comparable with that of GRB 170817A. Therefore, if GRBs are ten times as bright as GRB 170817A, CGBM can measure the spectral parameters of GRBs even if $E_{\text {peak }}$ of GRBs are as soft as $E_{\text {peak }}$ of GRB 170817A. 7 GRBs out of 15 which are the rest of the 19 short GRBs detected by the CGBM are also shown in Figure 5 based on the spectral parameters which were reported by Fermi-GBM to GCN circular [9]. The lowest flux GRB in Figure 5 was GRB 160726A of which flux was $\sim 1.2 \times 10^{-6} \mathrm{erg} \mathrm{cm}^{-2} \mathrm{~s}^{-1}$. Therefore, CGBM should detect GRBs more than $\sim 4$ times as bright as GRB 170817A. Furthermore, the CGBM detected 8 more short GRBs. While the SNRs of GRB 160726A measured by SGM in the $40 \sim 1000 \mathrm{keV}$ was 14 , that of $6 \mathrm{GRBs}$ out of 8 were distributed $9 \sim 12$.

\section{Summary}

CGBM has detected 161 GRBs which include 19 short bursts and 142 long bursts since October 2015. Although the ISS structures block some part of the field of view of the CGBM, CGBM 


\begin{tabular}{cccccccc}
\hline GRB name & CHXM1 & CSGM & $\alpha$ & $\begin{array}{c}E_{\text {peak }} \\
{[\mathrm{keV}]}\end{array}$ & averaged flux & $\chi^{2} /$ d.o.f & null hypothesis probability \\
\hline \hline $151225 \mathrm{~A}$ & $0.91_{-0.17}^{+0.20}$ & $1.18_{-0.21}^{+0.27}$ & $-1.05_{-0.28}^{+0.33}$ & $431_{-99}^{+167}$ & $(2.82 \pm 0.21) \times 10^{-6}$ & $57.82 / 55$ & 0.372 \\
$160709 \mathrm{~A}$ & $0.96_{-0.21}^{+0.27}$ & $1.36_{-0.26}^{+0.36}$ & $-0.49_{-0.20}^{+0.22}$ & $2053_{-325}^{+428}$ & $(6.86 \pm 0.47) \times 10^{-6}$ & $42.39 / 38$ & 0.287 \\
$180703 \mathrm{~B}$ & $1.05_{-0.09}^{+0.11}$ & $1.05_{-0.12}^{+0.14}$ & $-1.21_{-0.22}^{+0.24}$ & $146_{-17}^{+18}$ & $(3.27 \pm 0.13) \times 10^{-6}$ & $83.41 / 110$ & 0.972 \\
$190610 \mathrm{~A}$ & $1.02_{-0.19}^{+0.24}$ & $1.41_{-0.24}^{+0.31}$ & $-0.47_{-0.25}^{+0.28}$ & $792_{-126}^{+174}$ & $(5.83 . \pm 0.36) \times 10^{-6}$ & $59.11 / 57$ & 0.398 \\
\hline
\end{tabular}

Table 3: Spectral parameters of short GRBs detected by CGBM

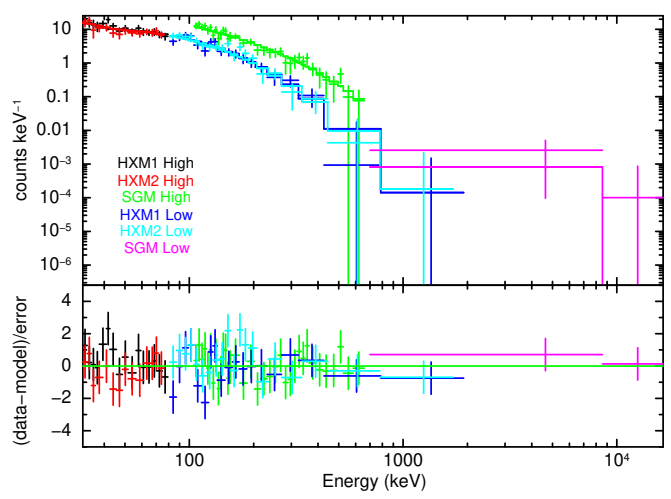

Figure 4: The spectrum of GRB 180703B with a cutoff power-law model. Points show CGBM data. Solid lines indicate the optimized cutoff power-law model.

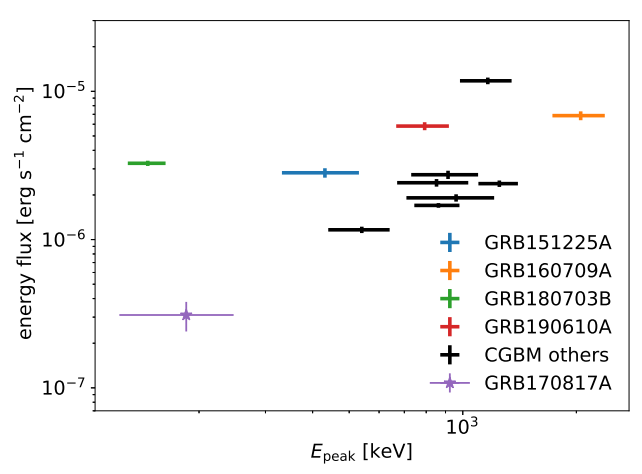

Figure 5: The distribution of energy flux (30 $1000 \mathrm{keV}$ ) vs. $E_{\text {peak }}$. Crosses show the points of short GRBs detected by CGBM. Star indicates the point of GRB 170817A based on the Fermi-GBM observation.

is observing GRBs with the wide field of view. We performed spectral analysis for 4 bright short GRBs detected by CGBM. We found the 4 bright GRB detected by CGBM are more than $\sim 10$ times as bright as that of GRB 170817A. We also found that the $E_{\text {peak }}$ of GRB $180703 \mathrm{~B}$ is comparable with that of GRB 170817A.

\section{References}

[1] P. Kumar, and B. Zhang, The physics of gamma-ray bursts \& relativistic jets, Physics Report, 561, 1, (2015).

[2] C. Kouveliotou et al., IDENTIFICATION OF TWO CLASSES OF GAMMA-RAY BURSTS, THE ASTROPHYSICAL JOURNAL, , 413, L101, (1993).

[3] T. J. Galama et al., An unusual supernova in the error box of the $\gamma$-ray burst of 25 April 1998, Nature, 395, 670, (1998).

[4] B. P. Abbott et al., GW170817: Observation of Gravitational Waves from a Binary Neutron Star Inspiral, PHYSICAL REVIEW LETTERS, 119, 161101, (2017).

[5] LIGO Scientific Collaboration and Virgo Collaboration, Fermi Gamma-ray Burst Monitor, and INTEGRAL Gravitational Waves and Gamma-Rays from a Binary Neutron Star Merger: GW170817 and GRB 170817A, THE ASTROPHYSICAL JOURNAL LETTERS, 848, L13, 27, (2017).

[6] S.Torii for the CALET Collaboration The CALorimetric Electron Telescope (CALET) on the ISS: Preliminary Results from On-orbit Observations since October, 2015, Proc.35th ICRC, 848, L13, 27 , (2017). 
[7] K. Yamaoka et al., The CALET Gamma-ray Burst Monitor (CGBM)) Proc. 7th Huntsville Gamma-Ray Burst Symposium (Nashville, USA, 2013), paper 41 in eConf Proceedings C1304143. (2013)

[8] NASA HEASARC battblocks https://heasarc.gsfc.nasa.gov/ftools/caldb/help/battblocks.html

[9] The Gamma-ray Coodinates Network (GCN), https: / /gcn.gsfc.nasa.gov/

[10] Fermi Trigger Information https://gcn.gsfc.nasa.gov/fermi_grbs.html

[11] D. Gruber et al., THE FERMI GBM GAMMA-RAY BURST SPECTRAL CATALOG: FOUR YEARS OF DATA, THE ASTROPHYSICAL JOURNAL SUPPLEMENT SERIES, 211, 12, 27pp, (2014).

[12] A. von Kienlin et al., THE SECOND FERMI GBM GAMMA-RAY BURST CATALOG: THE FIRST FOUR YEARS, THE ASTROPHYSICAL JOURNAL SUPPLEMENT SERIES, 211, 13, 13pp, (2014).

[13] P. N. Bhat et al., THE THIRD FERMI GBM GAMMA-RAY BURST CATALOG: THE FIRST SIX YEARS, THE ASTROPHYSICAL JOURNAL SUPPLEMENT SERIES, 223, 28, 18pp, (2016).

[14] The BATSE Current Gamma-Ray Burst Catalog, the BATSE GRB Team, https://gammaray.msfc.nasa.gov/batse/grb/catalog/current/

[15] The Swift/BAT Gamma-Ray Burst Catalog, https://swift.gsfc.nasa.gov/results/batgrbcat/

[16] S. Agostinelli et al., Geant4-a simulation toolkit, Nuclear Instruments and Methods in Physics Research A, 506, 250, (2003).

[17] NASA HEASARC Xspec, https://heasarc.gsfc.nasa.gov/xanadu/xspec/

[18] Y. Kawakubo, Systematic analysis of Gamma-ray bursts observed by CALET Gamma-ray Burst Monitor, Doctoral thesis, Aoyama Gakuin University, (2018).

[19] Y. Kawakubo et al., Detection of the thermal component in GRB 160107A Publ. Astron. Soc. Japan, 70 (1), 6, 1, (2018).

[20] R. Sari, T. Piran, \& R. Narayan, SPECTRA AND LIGHT CURVES OF GAMMA-RAY BURST AFTERGLOWS, THE ASTROPHYSICAL JOURNAL, 497, L17, (1998).

[21] A. Goldstein et al., An Ordinary Short Gamma-Ray Burst with Extraordinary Implications: Fermi-GBM Detection of GRB 170817A, THE ASTROPHYSICAL JOURNAL LETTERS, 848, L14, (2017). 